\title{
Obesity and gynecologic surgery via minimally invasive surgery with comparison to open laparotomy; a case report and review of the literature
}

\begin{abstract}
Background: Minimally invasive gynecological surgery (MIGS), has many benefits for patients, such as shorter recovery times and a decrease in postoperative pain. MIGS can prove challenging in obese patients as well. Obesity complicates MIGS due to the technical challenge for the surgeon as well as patient positioning. Often open laparotomy is done due to the aforementioned issues. However, MIGS provides better outcomes for patients.

Case: We report a case of a 37 year old G1P1 with a BMI of $80.72 \mathrm{~kg} / \mathrm{m} 2$ who complained of menorrhagia for 4 months. The patient was given a trial of OCP therapy for conservative treatment which did ameliorate the complaint. The patient continued to have heavy vaginal bleeding passage of large clots. Along with blood work, a pelvic ultra sound was done which showed bilateral multicystic and septated adnexal masses on the ovaries. A CT scan was also done which confirmed the mass, without a concern for malignancy. The patient was then scheduled for a total laparoscopic hysterectomy and bilateral salpingo-oophorectomy, via laparoscopic technique. The patient tolerated the procedure well. The adnexal masses were sent to pathology which revealed serous borderline tumors of the ovaries.

Conclusion: Gynecological surgery in the obese patient provides a challenge during the patient's pre, intra and postoperative management. Using MIGS, obese patients can have shorter hospital stay, with fewer complications as well as have less postoperative pain.
\end{abstract}

Keywords: complications, gynecologic surgery, minimally invasive surgery, obesity, MIGS, trendelenburg, BMI
Volume 10 Issue 2 - 2019

\begin{abstract}
Shadi Rezai,' Richard Giovane, ${ }^{2}$ Allison J Lazenby, ${ }^{3}$ Celestine S Tung, ${ }^{5}$ Cassandra E Henderson, ${ }^{4}$ Xiaoming Guan'

'Division of Minimally Invasive Gynecologic Surgery, Department of Obstetrics and Gynecology, Baylor College of Medicine, USA

${ }^{2}$ Department of Family Medicine, University of Alabama, USA ${ }^{3}$ The University of Alabama (UAB) School of Medicine, 1670 University Boulevard, USA

${ }^{4}$ Maternal and Fetal Medicine, Department of Obstetrics and Gynecology, Lincoln Medical and Mental Health Center, USA ${ }^{5}$ Division of Gynecologic Oncology, Department of Obstetrics and Gynecology, Baylor College of Medicine, USA
\end{abstract}

\begin{abstract}
Correspondence: Xiaoming Guan MD PhD, Section Chief and Fellowship Director, Division of Minimally Invasive Gynecologic Surgery, Department of Obstetrics and Gynecology, Baylor College of Medicine, 665I Main Street, 10th Floor, Houston, Texas, 77030, USA, Tel (832) 826-7464, Fax (832) 825-9349, Email xiaoming@bcm.edu
\end{abstract}

Received: March 03, 2019 | Published: April 25, 2019

\section{Background}

The benefits of minimally invasive techniques in gynecologic surgery are well-known and include faster recovery times, decreased postoperative pain, and faster return of bowel function. ${ }^{1}$ Barring contraindications, most physicians and patients elect to perform and undergo, minimally invasive surgery if available.

Obesity is one factor that complicates the decision to proceed with laparoscopic or robotic surgery. A minimally invasive procedure becomes more technically challenging for the surgeon in an obese patient. ${ }^{1}$ Concerns about patient positioning and optimal visualization during surgery lead to laparotomy in many obese patients. ${ }^{2}$ However, despite the adverse associated effects of obesity on the cardiovascular and respiratory systems, minimally invasive techniques may still be preferable over laparotomy to minimize postoperative complications in patients with compromised health status.

We discuss a case report and review the current literature comparing postoperative complications in minimally invasive gynecologic surgery versus laparotomy to advocate for the use of laparoscopic and robotic techniques in gynecologic surgery. We aim to highlight the important benefits of laparoscopic surgical techniques in patients regardless of Body Mass Index (BMI).

\section{Presentation of the case}

At the time of her surgery, the patient was a 37 -year-old G1P1 woman with a BMI of $80.72 \mathrm{~kg} / \mathrm{m}^{2}$ and a past medical history significant for menorrhagia, bilateral adnexal masses, hypertriglyceridemia, and lymphedema in the legs bilaterally.

She originally presented to the emergency room, complaining of menorrhagia for a few weeks and was given a norethindrone-ethinyl estradiol taper. She followed up with her PCP and reported the OCP taper slowed her bleeding somewhat. She was however referred to OBGYN at La Nueva Casa Clinic and was seen there on 6/12/14 complaining of continued heavy periods. She reported passing golf ball-sized clots and changing feminine hygiene products every two hours. Since menarche at the age of 10, her periods had been 3-7 days with regular flow except for one episode of heavy bleeding years prior which was treated with OCP taper. She reported missed periods in FebApril of the year of her presentation. The patient had associated hot flashes and constipation. She reported no vaginal discharge, itching, 
or swelling of labia. She exhibited no hirsutism or acne, had no nipple discharge, headaches or visual changes. She was going through a divorce and was not sexually active at the time. She had no history of abnormal pap smears, mammograms, or STIs; she had one caesarean section in 2001. The patient worked as a caregiver for elderly. Family history was unobtainable as she was adopted. She reported no used of tobacco, alcohol, or drugs. Review of systems was significant for weight loss, fatigue, shortness of breath and night sweats. She had a past history of an ovarian cyst noted in 2011.

Her medication list included furosemide, amitriptyline, levothyroxine, gabapentin, terbinafine, ibuprofen, phenazopyridine $\mathrm{HCl}$, nystatin, Norco, ferrous sulfate, cetirizine, naproxen, omega fatty acid supplement, and biotin.

At the time of her evaluation, her blood pressure was elevated at $141 / 83$; pulse was 82 , respiratory rate was 20 , and temperature was 98.3F. Physical exam showed a morbidly obese white female in no acute distress. Grape-sized blood clots were visualized in the vaginal vault; cervix was unable to be visualized and uterus and adnexa were unable to be palpated due to patient's habitus. Hemoglobin and hematocrit were low, at $7.3 \mathrm{~g} / \mathrm{dL}$ and $25.9 \%$, respectively. MCV was mildly elevated at $94 \mathrm{fL}$. TSH was also elevated at $3.87 \mathrm{uIU} / \mathrm{mL}$. Urine pregnancy test was negative.

The patient was scheduled for an ultrasound and FSH, estradiol, PL, DHEA-S, free testosterone, and 17-hydroxyprogesterone levels were ordered. The patient was advised to follow up with her primary care physician regarding her elevated TSH.

On 6/19/14, pelvic ultrasound showed large, bilateral multicystic and septated adnexal masses. The right mass (Figure 1) measured $13.7 \times 14 \times 8.8 \mathrm{~cm}$, while the left mass (Figure 2) measured $15.4 \times 12.2 \times 21.1 \mathrm{~cm}$. An enlarged uterus was also noted, but imaging was overall limited due to the patient's habitus.

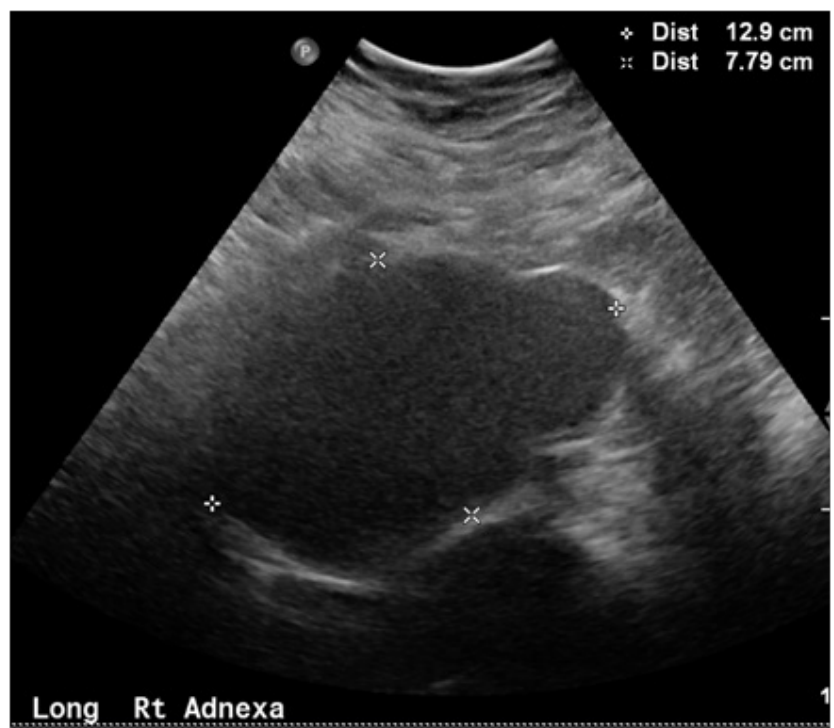

Figure I Pelvic ultrasound showing Multicystic and a septated right adnexa mass measuring $13.7 \times 14.0 \times 8.8 \mathrm{~cm}$.

The patient had a follow-up office visit for abnormal pelvic US results on 7/1/14. Previous lab-work showed decreased levels of FSH, LH, and estradiol, at $2.63 \mathrm{IU} / \mathrm{L}, 1.84 \mathrm{IU} / \mathrm{L}$, and $<11.8 \mathrm{pg} / \mathrm{mL}$, respectively. Results and need for surgery were discussed with patient.
CEA, CA-125, and CA 19-9 levels were ordered and the patient was referred to gynecologic oncology.

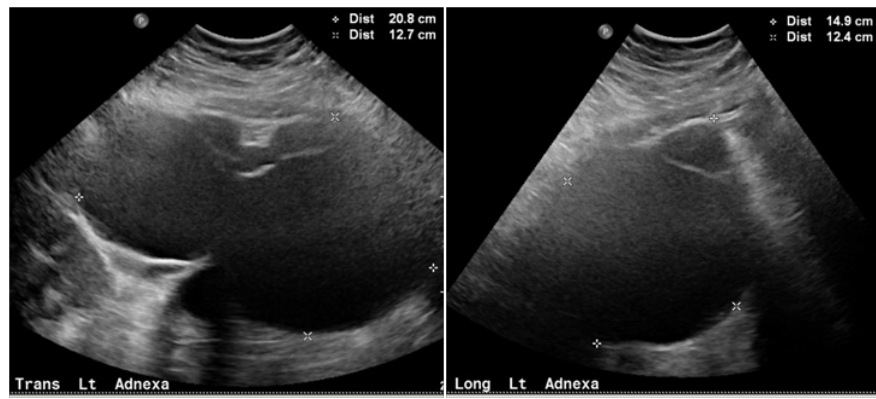

Figure 2 Pelvic ultrasound showing Multicystic and septated left adnexal mass measuring $15.4 \times 12.2 \times 21.1 \mathrm{~cm}$.

The patient subsequently had a CT scan on 7/23/14 which confirmed the presence of large bilateral ovarian masses, which were noted to be mostly solid and concerning for malignancy. No lymphadenopathy was noted. The also was found to have hepatic steatosis, diverticulosis, and a large cystic lesion of the right kidney.

Total laparoscopic hysterectomy and bilateral salpingooophorectomy were scheduled for $9 / 4 / 14$; the potential need for exploratory laparotomy due to her size was discussed with the patient. On $9 / 1 / 14$, the patient was admitted to hospital for a pre-op evaluation. Her CA-125 was noted to be elevated at $132.2 \mathrm{U} / \mathrm{mL}$. Physical exam was unremarkable. A type and cross as well as a $\mathrm{CBC}$ was ordered for the day of her surgery. It was also noted that the patient had a subclavian vessel pressing on her trachea and needed fiberoptic intubation, per anesthesiology.

On $9 / 4 / 14$, the patient was taken to OR for TLH and BSO as planned. She was appropriately positioned (Figure 3); the decision was made for a LUQ entry due to the patient's habitus and large size of ovarian masses. A trochar was placed at Palmer's point, the abdomen was insufflated with $\mathrm{CO}_{2}$, and ports were subsequently placed. There was some concern about laceration to liver during placement of the trochar, but the surgery continued with no signs of active bleeding. Visualization was difficult; both adnexal masses were found to be torsed. The LigaSure was used to suture ligate both ovarian masses, as well as the uterus and cervix. The uterus was placed in an EndoCatch bag and removed through the vagina. The adnexal masses were too large to be removed through the vagina; the decision was made to convert the supraumbilical port to a $7 \mathrm{~cm}$ handassisted port, with subsequent drainage and removal of each ovarian mass in a sterile X-ray cover bag. Both masses, uterus, and cervix were sent to pathology for frozen section. The vaginal cuff was closed and the bladder was inspected with a 30-degree cystoscope. The upper abdomen was again inspected due to initial concern of liver injury; general surgery was consulted for evaluation. A 1-cm through-andthrough injury to the left lobe of the liver, as well as injury to the anterior body of the stomach measuring approximately 5-10 mm, was found. The decision was made to convert to an exploratory laparotomy due to poor visualization of the injuries. The injury to the stomach was repaired by general surgery; the injury to the liver was hemostatic with no need for repair. The total estimated blood loss during the procedure was $2500 \mathrm{cc}$ and the patient was given two units of pRBCs. The abdomen was closed; the patient tolerated the procedure well. She remained intubated and was transferred to the SICU.

The patient was extubated on post-operative day 1 and remained 
in the SICU for two days. She had several episodes of desaturation during sleep which required use of a BiPAP machine and/or nasal cannula. She was discharged on post-operative day 9 on home oxygen.
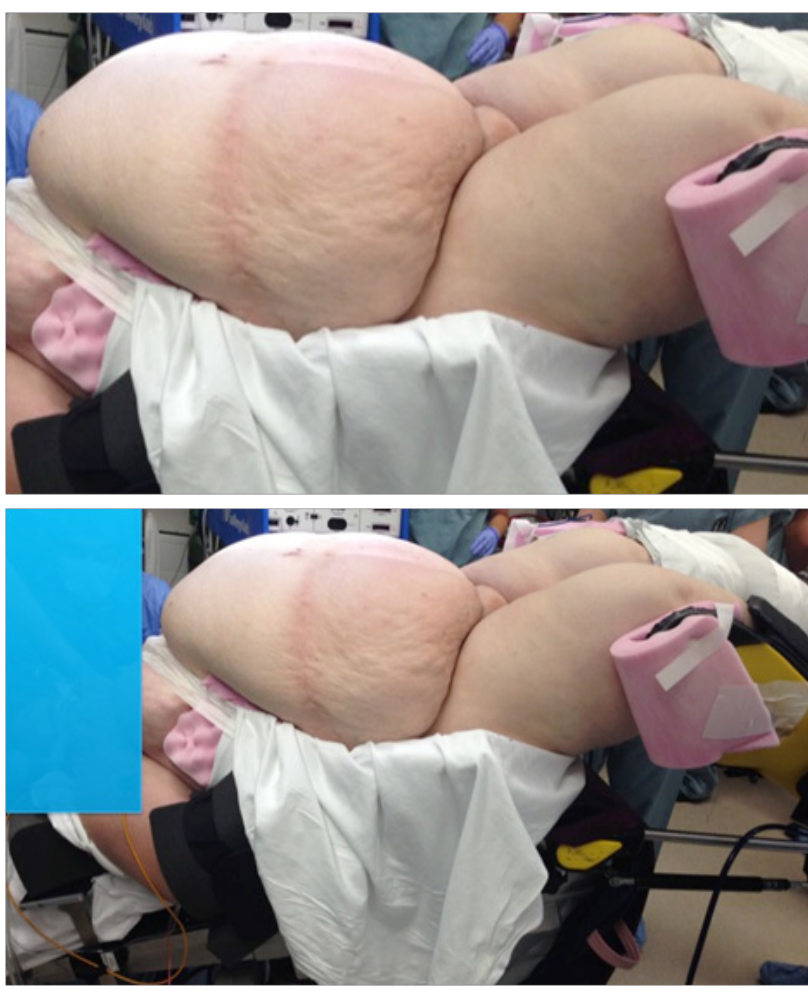

Figure 3 Positioning obese patients for minimally invasive gynecologic surgery.

Pathologic examination of the adnexal masses revealed serous borderline tumors of the ovaries, with involvement of the surface of each ovary. Stage was reported as 1C2.

The patient's postoperative course was otherwise unremarkable with normal wound healing. Follow-up visits were conducted at 3-4 month intervals.

\section{Discussion}

Obesity as a diagnosis is defined by three classes. Class 1 encompasses those with a BMI of 30-34.9, Class II of 35-39.9 and class III of a BMI greater than $40 .{ }^{3}$

Gynecological surgery in obese patients, defined as a BMI of greater than 30 , can pose a health risk to the patient during the operation and well as post-operatively. Intraoperatively, patients who are obese have an increase in oxygen consumption which can lead to a decrease in oxygenation during apnea. Moreover, patients with obesity are more prone to developing left and heart failure. ${ }^{4}$ Poster operatively, obese patient are at an increased risk of infection and venous thromboembolisms. ${ }^{5}$

Doing minimally invasive gynecology surgery [MIGS] versus a laparotomy approach in obese patients has advantages and disadvantages. MIGS has been shown to have a lower post-operative complication rate, lower hospital stays and less post-operative pain when compared to laparotomy (Table 1). ${ }^{6-9}$

It was found that MIGS in obese patients provided a safe approach for gynecological surgery. ${ }^{2,10,11}$ These studies showed that the complication was low and as reported by Wysham et al., ${ }^{2}$ complications can be as low as $3 \% .^{2}$ Although robotic surgery has its benefits of having fewer complications; there is the risk that the surgery might be converted to laparotomy. As reported by Cosin et al. ${ }^{12}$ the likelihood of converting a robotic surgery to laparotomy has a positive correlation with a patient's BMI. ${ }^{12}$ However, although there is a great chance to convert to laparotomy, if successful, hospital stay and morbidity of doing MIGS is the same as that of a patient with a normal BMI. ${ }^{13}$ When comparing MIGS versus laparotomy, Yu et al. ${ }^{6}$ reported that, patients who had laparotomy for gynecological oncology cases, had a longer hospital stay of 7 days as well as had an increased risk for developing infections at the surgical site. ${ }^{14,15}$

Proper positioning techniques for obese patients for gynecological surgery are important to minimize post-operative complications such as nerve damage. The most common nerve injuries are from ulnar and sciatic compressions due to excessive weight compressing these nerves as well as the patient being in a deep Trendelenburg position. ${ }^{6}, 16$ Common techniques to avoid nerve injury are to have the patient in the military position with their legs in the lithotomy position. ${ }^{17}$ This will minimize any excessive compression to nerves. Entry to the abdominal cavity also poses a challenge as the patient as an increased in fascia to abdominal wall. In a patient with a normal BMI, the trocar entry is usually at the umbilicus as it is the thinnest point of entry; however, in obese patients, the umbilicus moves more caudally and can prevent the surgeon from getting optimal viewing. ${ }^{18}$ In this regard, initial entry is usually done via the left upper quadrant or via supraumbilical region. This mitigates the risk of poor triangulation as well as obstructing proper view. ${ }^{6,19}$ Although obesity poses an increased risk that laparoscopy will be converted to laparotomy, certain techniques can be done peri-operatively to decrease this risk Using a steep Trendelenburg position sparingly can minimized the risk as it would prevent a decrease in minute ventilation of the patient; similarly, proper retraction within the abdominal wall has the same benefits. Lastly, a tilt test, while in Trendelenburg position should be done before an incision is made to assess the patient's ability to maintain adequate perfusion. ${ }^{19}$

Table I Postoperative Recovery and Complications for Minimally Invasive Gynecologic Surgery (MIGS) versus Open Surgical Technique (Laparotomy)

\begin{tabular}{|c|c|c|c|c|c|c|}
\hline Table I: Study & $\begin{array}{l}\text { Laparotomy } \\
\text { cases }\end{array}$ & $\begin{array}{l}\text { MIGS } \\
\text { cases }\end{array}$ & $\begin{array}{l}\text { Hospital stay for } \\
\text { laparotomy cases }\end{array}$ & $\begin{array}{l}\text { Hospital stay for MIGS } \\
\text { cases }\end{array}$ & $\begin{array}{l}\text { Complications for } \\
\text { laparotomy cases }\end{array}$ & $\begin{array}{l}\text { Complications for } \\
\text { MIGS cases }\end{array}$ \\
\hline Yu et al. ${ }^{6}$ & 4 & 4 & 11.5 & 4 & 4 & 0 \\
\hline Eisenhauer et al. ${ }^{7}$ & 154 & 57 & N/A & $N / A$ & Incidence:35\% & Incidence: $8 \%$ \\
\hline Walker et al. ${ }^{8}$ & 246 & 1248 & $\begin{array}{l}94 \% \text { [Greater than } \\
2 \text { days] }\end{array}$ & $52 \%$ [Greater than 2 days] & $21 \%$ & $14 \%$ \\
\hline Geppert et al. ${ }^{9}$ & 64 & 25 & 3.8 & 1.6 & $23 / 64=36 \%$ & $2 / 25=8 \%$ \\
\hline
\end{tabular}




\section{Conclusion}

Gynecological surgery in the obese patient provides a challenge during the patient's pre, intra and postoperative management. Using MIGS, obese patients can have shorter hospital stay, with fewer complications as well as have less post-operative pain. MIGS is a good alternative for gynecological surgery; however, care must be taken on proper positioning of the patient as well as the surgical approach to the abdominal cavity.

\section{Acknowledgments}

Dr. Xiaoming Guan is a speaker for Applied Medical, Rancho Santa Margarita, California.

\section{Conflicts of interest}

Other authors did not report any potential conflicts of interests.

\section{References}

1. Cooper K, Falcone T. Gynecologic surgery in the obese patient. J Minim Invasive Gynecol. 2014;21(2):155-156.

2. Wysham WZ, Kim KH, Roberts JM, et al. Obesity and perioperative pulmonary complications in robotic gynecologic surgery. Am J Obstet Gynecol. 2015;213(1):33.e1-33.e7.

3. Kabon B, Nagele A, Reddy D, et al. Obesity decreases perioperative tissue oxygenation. Anesthesiology. 2004;100(2):274-280.

4. Executive summary of the clinical guidelines on the identification, evaluation, and treatment of overweight and obesity in adults. Arch Intern Med. 1998;158(17):1855-1867.

5. Committee opinion no. 619: Gynecologic surgery in the obese woman Obstet Gynecol. 2015;125(1):274-278.

6. Yu CK, Cutner A, Mould T, et al. Total laparoscopic hysterectomy as a primary surgical treatment for endometrial cancer in morbidly obese women. BJOG. 2005;112(1):115-117.

7. Eisenhauer EL, Wypych KA, Mehrara BJ, et al. Comparing surgical outcomes in obese women undergoing laparotomy, laparoscopy, or laparotomy with panniculectomy for the staging of uterine malignancy. Ann Surg Oncol. 2007;14(8):2384-2391.
8. Walker JL, Piedmonte MR, Spirtos NM, et al. Recurrence and survival after random assignment to laparoscopy versus laparotomy for comprehensive surgical staging of uterine cancer: Gynecologic Oncology Group LAP2 Study. J Clin Oncol. 2012;30(7):695-700.

9. Geppert B, Lonnerfors C, Persson J. Robot-assisted laparoscopic hysterectomy in obese and morbidly obese women: surgical technique and comparison with open surgery. Acta Obstet Gynecol Scand. 2011;90(11):1210-1217

10. Kannisto P, Harter P, Heitz F, et al. Implementation of robot-assisted gynecologic surgery for patients with low and high BMI in a German gynecological cancer center, Arch Gynecol Obstet. 2014;290(1):143-148.

11. Scheib SA, Tanner E, Green IC, et al. Laparoscopy in the morbidly obese: physiologic considerations and surgical techniques to optimize success. $J$ Minim Invasive Gynecol. 2014;21(2):182-195.

12. Cosin JA, Brett Sutherland MA, Westgate CT, et al. Complications of Robotic Gynecologic Surgery in the Severely Morbidly Obese. Ann Surg Oncol. 2016;23(12):4035-4041.

13. Peijnenburg E, Tanner EJ, Stone RL, et al. Laparoscopic Abdominal Access Using a Modified Left Upper Quadrant Technique in Morbidly Obese Women Undergoing Gynecologic Surgery. J Minim Invasive Gynecol. 2015;22(6S):S197.

14. Peng J, Sinasac S, Pulman KJ, et al. The Feasibility of Laparoscopic Surgery in Gynecologic Oncology for Obese and Morbidly Obese Patients. Int J Gynecol Cancer. 2018;28(5):967-974.

15. Eltabbakh GH, Piver MS, Hempling RE, et al. Laparoscopic surgery in obese women. Obstet Gynecol. 1999;94(5Pt 1):704-708.

16. Lamvu G, Zolnoun D, Boggess J,et al. Obesity: physiologic changes and challenges during laparoscopy. Am J Obstet Gynecol. 2004;191(2):669674.

17. Hurd WW, Bude RO, Delancey JO, et al. The relationship of the umbilicus to the aortic bifurcation: implications for laparoscopic technique. Obstet Gynecol. 1992;80(1):48-51.

18. Afors K, Centini G, Murtada R, et al. Obesity in laparoscopic surgery. Best Pract Res Clin Obstet Gynaecol. 2015;29(4):554-564.

19. Stany MP, Winter WE, Dainty L, et al. Laparoscopic exposure in obese high-risk patients with mechanical displacement of the abdominal wall. Obstet Gynecol. 2004;103(2):383-386. 